\title{
Aldehyde dehydrogenase
}

An enzyme with two distinct catalytic activities at a single type of active site

\author{
R. Julian S. DUNCAN \\ The Wellcome Research Laboratories, Beckenham, Kent BR3 3BS, U.K.
}

(Received 4 March 1985/29 April 1985; accepted 7 May 1985)

\begin{abstract}
The evidence for and against the esterase and dehydrogenase active sites of aldehyde dehydrogenase being topologically distinct is examined. It is found that all the evidence (including all that previously amassed by others in favour of distinct binding domains) is actually consistent with, and in favour of, a single type of catalytic site having both activities. The existence of separate high- $K_{\mathrm{m}}$ modulating sites for the enzyme is also questioned.
\end{abstract}

Aldehyde dehydrogenases from a variety of mammalian species are enzymes that exhibit two catalytic activities, namely the eponymous aldehyde oxidation and an esterase activity towards 4nitrophenyl esters (Feldman \& Weiner, 1972; Sidhu \& Blair, 1975a,b; Duncan, 1977; MacGibbon et al., 1978). There is general agreement that dehydrogenation of aldehydes in concentrations up to $50-100 \mu \mathrm{M}$ by these enzymes follows a compulsory-order reaction pathway: coenzyme binding first followed by aldehyde, then irreversible release of the acid product, and finally dissociation of the reduced coenzyme from the enzyme (Duncan \& Tipton, 1971 $b$; Feldman \& Weiner, 1972; MacGibbon et al., 1977; Hart \& Dickinson, 1982). The following comments refer to aldehyde dehydrogenase in general, but specifically to the enzymes from pig brain and rabbit liver (Duncan \& Tipton, $1971 a, b$; Duncan, 1977, 1979) and from sheep liver (MacGibbon et al., 1977, 1978; Kitson, 1978, 1981, $1982 a, b ;$ Hart \& Dickinson, 1978, 1982; Agnew et al., 1981; Buckley \& Dunn, 1982; Blackwell et al., $1983 a, b$; Dickinson, 1985). A complication of this ordered mechanism is substrate activation, which is observed with some isoenzymes and acetaldehyde, propionaldehyde and (in some species) butyraldehyde (Duncan \& Tipton, 1971b; MacGibbon et al., 1977; Hart \& Dickinson, 1982). The mechanism of activation is not clear, but for the sheep liver cytosolic enzyme Hart \& Dickinson (1982) proposed on kinetic grounds that an enzyme $\mathrm{NADH} \cdot$ aldehyde ternary complex is formed at high (millimolar) concentrations of aldehyde and that dissociation of NADH from this complex is more rapid than from the enzyme. NADH binary complex (thought to be normally at least partly rate-determining in the ordered pathway). Direct spectrophotofluorimetric evidence for the occurrence of such a ternary complex has been presented along with a probable rate constant for its dissociation sufficient to explain the activation of the overall reaction (Dickinson, 1985). Blackwell et al. (1983a) explain the activation in terms of the existence of a modifier domain containing a low-affinity propionaldehyde-binding site (called $\mathrm{P} 2, K_{\mathrm{m}} 3.5 \mathrm{~mm}$; and spatially distinct from the catalytic aldehyde-binding domain P1, $\left.K_{\mathrm{m}} 1.1 \mu \mathrm{M}\right)$. Occupance of the low-affinity domain, at high aldehyde concentrations, leads to activation of the sheep liver cytosolic enzyme.

Hydrolysis of 4-nitrophenyl esters by aldehyde dehydrogenases occurs in the presence or in the absence of NAD; at $\mathrm{pH}$ values near neutrality ester hydrolysis is stimulated by both $\mathrm{NAD}^{+}(3-5-$ fold) and NADH (2-fold) (Feldman \& Weiner, 1972; MacGibbon et al., 1978; Duncan, 1979), although at pH9.0 NADH inhibits in a partially competitive manner ester hydrolysis by a rabbit liver cytosolic aldehyde dehydrogenase. There are hence kinetic reasons for postulating the formation, under appropriate conditions, of a complex between enzyme, NADH and 4-nitrophenyl acetate.

\section{Theory}

\section{In favour of a single type of catalytic site}

It is known that at least two thiol groups occur close to the active site of aldehyde dehydrogenase, modification of one or more of which causes inhibition of activity (Duncan \& Tipton, 1971a; 
Duncan, 1979), and that certain thiol groups of the enzyme are chemically very reactive relative to most other thiol groups (Kitson, 1981). As has long been assumed by analogy with the mechanism (Harris \& Waters, 1976) of glyceraldehyde-3phosphate dehydrogenase, it is now known that an enzyme-thioester-NADH complex is formed during oxidation of certain aldehydes (Buckley \& Dunn, 1982; Dickinson, 1985). There are possible ramifications of this idea relating to the variety of interactions between thiol-modifying reagents and the enzyme, which are discussed by Kitson (1982a), but it has commonly been assumed that hydrolysis of 4-nitrophenyl esters occurs via acylation of those same reactive active-site thiol groups as are involved in the oxidation of aldehydes (Feldman \& Weiner, 1972; Sidhu \& Blair, 1975a,b; Eckfeldt \& Yonetani, 1976; Duncan, 1977).

If the assumption of commonality of the catalytic groups for esterase and dehydrogenase activities were true, then ester hydrolysis occurring in the presence of NADH should involve an enzyme-thioester - NADH central complex identical (except probably for enzyme isomerism) with that formed during aldehyde dehydrogenation. These considerations led to the proposal that it should be possible to reverse part of the dehydrogenase reaction pathway and produce acetaldehyde and $\mathrm{NAD}^{+}$during the hydrolysis of 4nitrophenyl acetate in the presence of NADH (Duncan, 1977). Subsequently Hart \& Dickinson (1978) showed that sheep liver cytosolic aldehyde dehydrogenase could be made to yield acetaldehyde or butyraldehyde if acylated with acetic anhydride or butyric anhydride in the presence of NADH. (This procedure would be expected to lead to the crucial enzyme-thioester-NADH complex). A determination of $\mathrm{NAD}^{+}$and aldehyde production during the hydrolysis by rabbit liver cytosolic aldehyde dehydrogenase of 4-nitrophenyl acetate in the presence of NADH showed that approx. $2 \%$ of hydrolytic cycles at $\mathrm{pH} 7.0$ gave rise to aldehyde and $\mathrm{NAD}^{+}$with correct stoicheiometry (Duncan, 1979). It is to be noted that the aldehyde dehydrogenase from Vibrio harveyi also can be reversed if acylated with long-chain $\left(\mathrm{C}_{14}\right)$ acyl$\mathrm{CoA}$, and that this enzyme has both dehydrogenase and esterase activities (Byers \& Meighen, 1984). This direct evidence makes the conclusion inescapable that the dehydrogenase and esterase activities of rabbit liver (and probably also sheep liver) cytosolic aldehyde dehydrogenase reside in the same catalytic region. Further, given the probable presence of a reactive thiol group at or near this active centre, the formation of a thioester during aldehyde oxidation, and the thiol-acylating ability of both acid anhydrides and 4-nitrophenyl acetate, it is logical to propose that at least one of the catalytic groups involved is the same for both the dehydrogenase and the esterase activities.

\section{Evidence for different catalytic sites}

Blackwell, Buckley and their colleagues believe that the esterase and dehydrogenase activities of the cytosolic aldehyde dehydrogenase from sheep liver are located at different catalytic domains and utilize different catalytic groups (MacGibbon et al., 1978; Blackwell et al., 1983a,b). A number of enzymes are known to catalyse different reactions at different active sites (Sakakibara et al., 1984; Eccleston et al., 1979), and so the data suggesting that aldehyde dehydrogenase should be classified as a member of that group of enzymes must be evaluated carefully. The published evidence upon which MacGibbon et al. (1978) and Blackwell et al. $(1983 a, b)$ have based their belief is, or has been, as follows: (i) inhibition of the esterase activity but not the dehydrogenase by high concentrations of $\mathrm{NAD}^{+}$(MacGibbon et al., 1978); (ii) inhibition of the dehydrogenase activity but not the esterase by disulfiram (Kitson, 1978; see MacGibbon et al., 1978); (iii) the partially competitive nature of the inhibition of the esterase activity by propionaldehyde at low ('P1-site' binding) concentrations, with fully competitive inhibition by high ('P2-site' binding) concentrations (Blackwell et al., 1983a); (iv) the (partially) non-competitive inhibition of esterase but competitive inhibition of dehydrogenase by chloral hydrate in the presence of $\mathrm{NAD}^{+}$, and the competitive inhibition of the esterase by chloral hydrate in the absence of NAD ${ }^{+}$(Blackwell et al., 1983a,b); (v) differential inhibition of the two activities by glyoxylate (mixed for the dehydrogenase at 'Pl' propionaldehyde concentrations, competitive for the esterase in the presence of $\mathrm{NAD}^{+}$; complex but probably competitive inhibition of the dehydrogenase in the presence of ' $\mathrm{P} 2$ ' propionaldehyde concentrations; the various $K_{\mathrm{i}}$ values are all of the same magnitude) (Blackwell et $a l ., 1983 a, b)$; (vi) some pre-steady-state results and metal-ion inhibition data that are adduced as evidence that the kinetic rate constants of esterase and dehydrogenase are different, although not necessarily involving different catalytic groups (Blackwell et al., 1983a).

\section{Analysis of the evidence for distinct catalytic domains}

(i) $\mathrm{NAD}^{+}$inhibition. This claim has been retracted (Agnew et al., 1981). Moreover, it is unnecessary to invoke a second (modifier) binding site for NAD, as proposed by MacGibbon et al. (1978), to explain inhibition by high concentrations of the coenzyme. Haldane (1930) has shown that such inhibition may arise by partial combination of two or more molecules of substrate at the normal active site, and extension of this to 
the observed inhibition of ester hydrolysis and aldehyde oxidation is straightforward.

(ii) Differential inhibition of the two activities by disulfiram has been shown to be a methodological artifact (Kitson, 1982a) due to protection of the enzyme mixed with 4-nitrophenyl acetate before the addition of disulfiram, but lack of protection by previous mixing with $\mathrm{NAD}^{+}$plus acetaldehyde. In the absence of substrates disulfiram inhibited both activities to essentially the same extent (Kitson, $1982 a$ ), in agreement with observation that iodoacetamide inhibited both activities of the rabbit liver cytosolic enzyme in parallel (Duncan, 1979). The lack of protection by aldehyde plus $\mathrm{NAD}^{+}$was ascribed by Kitson (1982a) to the known ability of disulfiram to react rapidly with the enzyme. NADH binary complex formed during the dehydrogenase catalytic cycle, whereas protection in the presence of saturating concentrations of 4nitrophenyl acetate was afforded because the enzyme would be always in (thiol-protecting) enzyme-ester or enzyme-acyl complexes. The informative experiment of mixing enzyme with 4nitrophenyl acetate before disulfiram and then investigating protection of the dehydrogenase was apparently not performed. It is, however, likely that protection would have been afforded, as preincubation with chloral hydrate in the absence of $\mathrm{NAD}^{+}$gave significant protection of the esterase against disulfiram (see also below), whereas indol-3-ylacetaldehyde (in the absence, but not in the presence, of $\mathrm{NAD}^{+}$) gave significant protection of the dehydrogenase (Kitson, 1982a). The results of crossed-protection experiments with these reagents were not reported.

Kitson (1978) showed that modification of sheep liver cytosolic aldehyde dehydrogenase with 2,2'-dithiodipyridine activated the dehydrogenase, but only in the presence of $\mathrm{NAD}^{+}$. The esterase activity was inhibited by this reagent, more completely in the presence of $\mathrm{NAD}^{+}$.
Similarly it has been shown that diethylstilboestrol activates the dehydrogenase and inhibits the esterase activities of rabbit liver (Duncan, 1977) and sheep liver (Kitson, 1982b) cytosolic aldehyde dehydrogenases. These differences can be accommodated in a kinetic scheme in which the two activities are functions of the same catalytic groups, but the reagents produce a new form of the enzyme having modified catalytic properties relative to the native enzyme (Duncan, 1977).

(iii) Partial competitive inhibition of the esterase activity by low ('Pl-site' binding) concentrations of propionaldehyde is in fact fully in accord with the hydrolysis of 4-nitrophenyl acetate and dehydrogenation of the aldehyde occuring at the same catalytic groups. The established ordered dehydrogenase pathway of the enzyme is shown in Scheme 1, with the enzyme forms known to bind and hydrolyse the ester also shown. The Scheme ignores isomeric enzyme forms, but these are, of course, indistinguishable by steady-state kinetics and do not affect the interpretation. With saturating $\mathrm{NAD}^{+}$, in the presence of 4-nitrophenyl acetate (the situation discussed by Blackwell et al., 1983a) the enzyme will be distributed between $\mathrm{E}_{\mathrm{NAD}^{+}}$and enzyme forms also involving the ester or its components. Propionaldehyde (at 'P1' concentrations) will bind only to the $\mathrm{E}_{\mathrm{NAD}^{+}}$binary complex.

This will occur at non-saturating ester concentrations (when $\mathrm{E}_{\mathrm{NAD}^{+}}$is present in kinetically significant concentrations), but not at saturating ester concentrations (when any free $\mathrm{E}_{\mathrm{NAD}^{+}}$will immediately react with ester), and hence inhibition by aldehyde can be overcome at saturating ester concentrations: i.e. the inhibition is competitive. However, at fixed non-saturating ester concentrations some of the $\mathrm{E}_{\mathrm{NAD}^{+}}$then present will be converted by aldehyde, via the ternary complex,

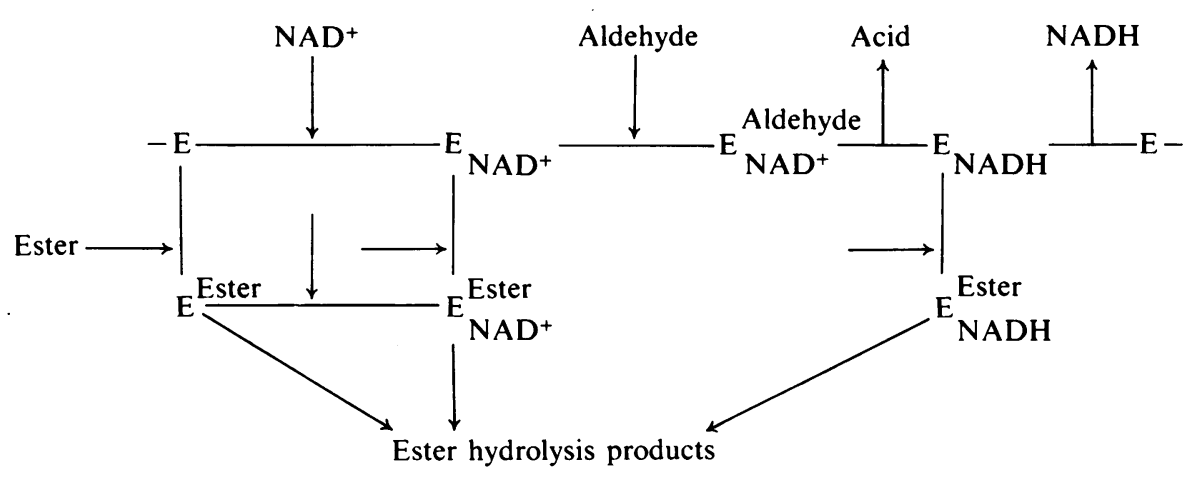

Scheme 1. Kinetic scheme for aldehyde dehydrogenation and ester hydrolysis, consistent with the experimental evidence, and requiring only one type of active site for aldehyde dehydrogenase 
into $\mathrm{E}_{\mathrm{NADH}}$. This complex will also bind and hydrolyse the ester, so that even at saturating aldehyde concentrations (when any $\mathrm{E}_{\mathrm{NAD}^{+}}$will be prevented from binding ester because of immediate reaction with aldehyde) hydrolysis of ester will still occur: i.e. inhibition is partial, as well as competitive. This result can be obtained equally well by derivation of the full rate equation or by inspection and application of the rules of Cleland (1970). A single active site acting on both dehydrogenase and esterase is hence fully compatible with Fig. 1 of Blackwell et al. (1983a) (wherein the concentration of aldehyde does not exceed $100 \mu \mathrm{M})$. Indeed, this type of inhibition is a necessity if only one type of active site is present. A complete analysis of the various interactions is given as Table 1.

Inhibition of the esterase by high ('P2-site' binding) concentrations of propionaldehyde is linear competitive in the absence of coenzyme (Blackwell et al., 1983a). This implies that at high concentrations of propionaldehyde ester and aldehyde binding are mutually exclusive, not shown in Scheme 1 (see below for a discussion of the kinetic implications of competitive inhibition). There are two explanations consistent with this finding, neither of which requires separate esterase and dehydrogenase catalytic sites. Either a separate 'P2 site' exists, which when occupied prevents access of 4-nitrophenyl esters to the dehydrogenase/esterase active site, but does not prevent access of aldehyde in the presence of $\mathrm{NAD}^{+}$(the effect on ester hydrolysis of this concentration of propionaldehyde, in the presence of $\mathrm{NAD}^{+}$, would also be competitive), or high concentrations of propionaldehyde bind to the normal dehydrogenase/esterase active site in the absence of NAD. In the latter case the dehydrogenase reaction must occur by an altered kinetic pathway at high concentrations of aldehyde, as proposed in detail by Hart \& Dickinson (1982) and by Sidhu \& Blair (1975b). It might be objected that, if such high concentrations of aldehyde do bind 'out of order' at the normal active site, then Kitson (1982a) should have seen protection in his experiments with disulfiram, as he used $1 \mathrm{~mm}$-acetaldehyde. According to Dickinson (1985), this concentration of propionaldehyde binds to the enzyme NADH complex, presumably (although not explicitly stated) at the normal aldehyde-binding site, and hence all forms of the enzyme might be expected to be protected (see Scheme $3 b$ ). The lack of protection may be rationalized by realizing that with a single aldehyde-binding site per catalytic unit an enzyme. $\mathrm{NADH}$ complex must be formed transiently during the catalytic cycle even in the presence of very high concentrations of aldehyde, and that this enzyme species may then react with either aldehyde or disulfiram. The reaction with disulfiram, even at micromolar concentrations, is extraordinarily rapid (Kitson, 1981) and may outpace the protective binding of acetaldehyde. A prediction from this mechanism is that high concentrations of propionaldehyde, in the absence of $\mathrm{NAD}^{+}$, should protect both esterase and dehydrogenase activities against disulfiram. Binding at a separate 'P2 site' in the absence of $\mathrm{NAD}^{+}$may or may not give protection.

(iv) The observations on the inhibition by chloral hydrate are perfectly consistent with a single catalytic site for both esterase and dehydrogenase. The statement in Blackwell et al. (1983b) 'In the presence of $\mathrm{NAD}^{+}$, chloral hydrate must bind in the $\mathrm{Pl}$ site since it is a competitive inhibitor of the dehydrogenase activity' is wrong. Competitive inhibition between two ligands does not indicate that the binding sites of those ligands are identical or even spatially close on the enzyme. Competitive inhibition merely indicates that binding of the

Table 1. Inhibitory interactions between aldehyde and 4-nitrophenyl esters

A single esterase/dehydrogenase active site is assumed with kinetic mechanism as in Scheme 1 or Scheme $3(b)$.

Aldehyde concentration Type of inhibition

1. Action of aldehydes on esterase activity

(a) $\mathrm{No} \mathrm{NAD+}$

\begin{tabular}{|c|c|}
\hline $\begin{array}{l}\text { Low } \\
\text { High }\end{array}$ & $\begin{array}{l}\text { None } \\
\text { Competitive, linear }\end{array}$ \\
\hline Low & $\begin{array}{l}\text { Competitive, pat } \\
\text { tial }\end{array}$ \\
\hline $\mathrm{High}$ & Competitive, linear \\
\hline
\end{tabular}

2. Action of 4-nitrophenyl esters on dehydrogenase activity $\left(\mathrm{NAD}^{+}\right.$saturating or non-saturating)

$\begin{array}{ll}\text { Low } & \text { Mixed, linear } \\ \text { High } & \text { Competitive, linear }\end{array}$


Hence chloral hydrate may or may not bind at the aldehyde-binding site; steady-state kinetics can give no further information on the point. Scheme 2 , which is an elementary extension of Scheme 1, shows an enzyme reaction pathway with identical esterase and dehydrogenase catalytic groups that, according to the rules of Cleland (1970) or by inspection, fits the inhibition data presented. There is an inconsistency in the data concerning inhibition of the esterase by chloral hydrate in the presence of $\mathrm{NAD}^{+}$[simple linear non-competitive inhibition in Blackwell et al. (1983b), but 'The inhibition pattern appeared to be mixed, with a linear slope replot and a hyperbolic intercept replot' (Blackwell et al., 1983a)]. Whichever is correct can be accommodated by Scheme 2, depending on whether the $\mathrm{E} \cdot \mathrm{NAD}^{+} \cdot$ chloral hydrate-ester complex hydrolyses the ester (mixed; non-linear) or not (non-competitive, with appropriate rate constants; linear). Scheme 2 further predicts mixed (or non-competitive) inhibition by chloral hydrate of aldehyde dehydrogenation (relative to aldehyde) at non-saturating coenzyme concentrations, in agreement with Sidhu \& Blair $(1975 b)$. The protection of esterase against disulfiram by chloral hydrate, as reported by Kitson $(1982 a)$, is, of course, to be expected from the mechanism of Scheme 2, but gives no information about where on the enzyme chloral hydrate binds, at the active site or away from it but giving protection by modulation of the protein's structure. Thus Fig. 2 of Blackwell et al. (1983a) is in full accord with the esterase and dehydrogenase activities being catalysed by the same active site.

It could be objected that binding of 4-nitrophenyl acetate to the enzyme and to the enzyme $\cdot$ chloral hydrate $\cdot \mathrm{NAD}^{+}$complex but not to the enzyme - chloral hydrate complex is unlikely. However, it is known that $\mathrm{NAD}^{+}$binding must alter the configuration of the enzyme in allowing access of the substrate aldehyde to the active site at low aldehyde concentrations, and so the proposed scheme is thermodynamically acceptable. Similar reasoning applies to the mutually exclusive binding of aldehyde or chloral hydrate to the enzyme. $\mathrm{NAD}^{+}$complex without exclusion of the ester by chloral hydrate: the aldehyde cannot bind in the absence of $\mathrm{NAD}^{+}$, but the ester can, and so the binding requirements, but not the site, for propionaldehyde and ester are necessarily different.

(v) The glyoxylate inhibition data are fully consistent with the esterase and dehydrogenase activities being catalysed at the same active site. Scheme 3(a) (another elementary extension of Scheme 1) leads directly to an expectation of inhibition of the form described (Blackwell et al., $1983 a$ ), as can be seen by application of the rules of Cleland (1970). The inhibition pattern observed at high propionaldehyde concentrations is consistent with Scheme $3(b)$. This (following the second mechanism discussed in the interactions between the enzyme and high concentrations of aldehyde above, but having little bearing on whether the esterase and dehydrogenase activities are catalysed at the same site except as discussed above) supposes out-of-order binding of aldehyde [on the basis of the proposals of Hart \& Dickinson (1982) and Dickinson (1985)] and binding of glyoxylate by only the enzyme $\cdot$ coenzyme complex produced transiently during the reaction. The nonlinearity with reciprocal aldehyde concentration arises from concurrent operation of the mechanism of Scheme 3(a) by way of some of the free enzyme that may arise. Both Scheme 3(a) and Scheme 3(b)



Scheme 2. Diagram of the interactions between chloral hydrate, aldehyde dehydrogenation and ester hydrolysis, assuming only one type of active site for the enzyme 


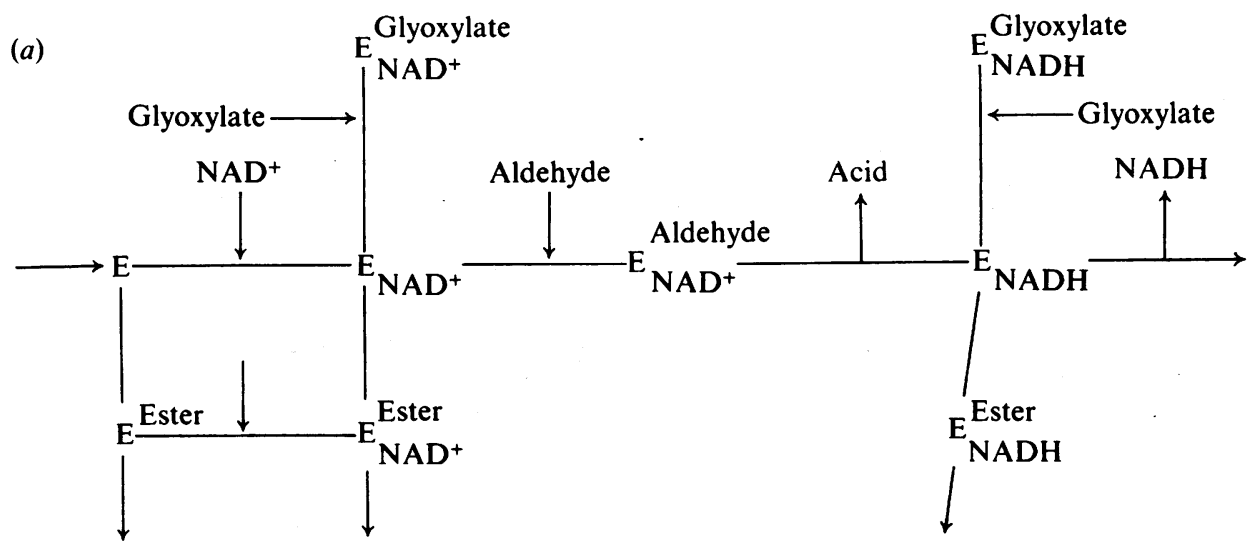

(b)

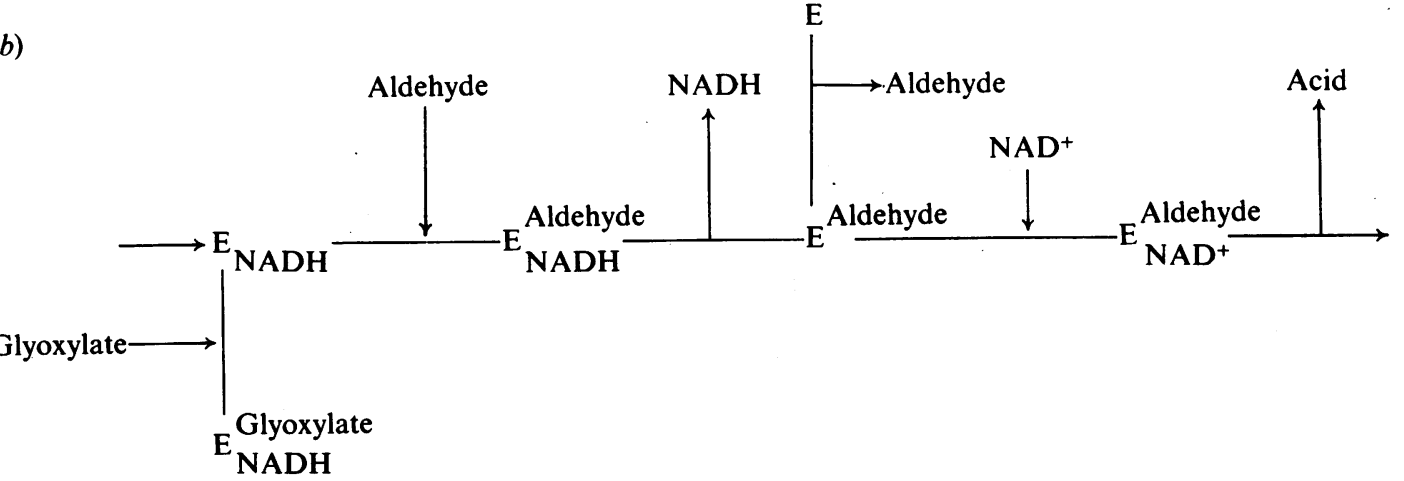

Scheme 3. (a) Diagram of the interactions between glyoxylate, aldehyde dehydrogenation and ester hydrolysis at relatively low aldehyde concentrations, and $(b)$ diagram of the interactions between glyoxylate and aldehyde dehydrogenation, consistent with the experimental evidence, at relatively high aldehyde concentrations

require that glyoxylate binds only to the enzyme. coenzyme complex, and hence are consistent with the details of the lag phase (hysteresis) noted by Blackwell et al. $(1983 b)$ when the enzyme was preincubated with $\mathrm{NAD}^{+}$and glyoxylate before initiation of the reaction.

If the presence of a modulator ('P2') site is allowed, rather than out-of-order binding, then the data are consistent with binding of glyoxylate to the enzyme-coenzyme complex causing exclusion of aldehyde from the ' $\mathrm{P} 2$ ' binding site [either by binding at that site or elsewhere on the enzyme, and not necessarily as supposed by Blackwell et al. $(1983 b)$; see the comments above about mutual exclusion and competitive inhibition].

(vi) It is agreed by Blackwell et al. (1983a) that the pre-steady-state kinetic differences between esterase and dehydrogenase activities provide evidence neither for nor against both reactions involving acylation of the same catalytic groups; they are not in any way inconsistent with the identity of the sites. It might be expected that the magnitude of the rate constants governing apparently identical reactions (e.g. deacylation of the enzyme $\cdot \mathrm{NADH} \cdot$ acyl complex formed either by the dehydrogenase pathway or by the esterase pathway in the presence of NADH) would be different, because not only are such intermediates formed by distinct routes leading to potentially dissimilar enzyme isomeric structures for the same complex, but also Katz \& Westley (1979) have shown that the nature of the first leaving group in modified enzyme (ping-pong) kinetic pathways can influence the kinetics of the second (recipient) substrate reaction.

\section{Conclusions}

There is no evidence that the esterase and dehydrogenase catalytic sites of aldehyde dehydrogenase are topologically distinct, but there is convincing evidence that the activities are catalysed by the same active centre, including all the evidence previously amassed in order to show the sites to be distinct. Under these circumstances the arguments advanced by Blackwell et al. (1983a) concerning the relationship between the 'P2 domain' and the esterase site are superfluous. 
Further, since the 'Pl domain' and the esterase site are, by all the evidence and argument presented here, identical, and, since Blackwell et al. (1983a) believe that the esterase binding domain is at the 'P2 domain', then the 'P1' and 'P2' domains must be identical also. It appears that the evidence for the 'P2 domain' should be re-evaluated in the light of this possibility: the hypothesis being that the 'P2' site is the 'P1' site in terms of the enzyme's structure but whose occupance in the absence of $\mathrm{NAD}^{+}$, or before $\mathrm{NAD}^{+}$during the catalytic cycle, leads to the modified kinetic, pre-steady-state and subunit interactions reported.

\section{References}

Agnew, K. E. M., Bennett, A. F., Crow, K. E., Greenway, R. M., Blackwell, L. F. \& Buckley, P. D. (1981) Eur. J. Biochem. 119, 79-84

Blackwell, L. F., Bennett, A. F. \& Buckley, P. D. (1983a) Biochemistry 22, 3784-3791

Blackwell, L. F., Bennett, A. F., Crow, K. E., Buckley, P. D. \& Deady, L. W. (1983b) Pharmacol. Biochem. Behav. 18, Suppl. 1, 83-87

Buckley, P. D. \& Dunn, M. F. (1982) Prog. Clin. Biol. Res. 114, 23-25

Byers, D. \& Meighen, E. (1984) J. Biol. Chem. 259, 71097114

Cleland, W. W. (1970) Enzymes 3rd Ed. 2, 1-61

Dickinson, F. M. (1985) Biochem J. 225, 159-165

Dixon, M. \& Webb, E. C. (1979) Enzymes, 3rd edn., p. 335, Longmans, London

Duncan, R. J. S. (1977) Biochem. J. 161, 123-130
Duncan, R. J. S. (1979) Biochem. J. 183, 459-462

Duncan, R. J. S. \& Tipton, K. F. (1971a) Eur. J. Biochem. 22, 257-262

Duncan, R. J. S. \& Tipton, K. F. (1971b) Eur. J. Biochem. 22, 538-543

Eccleston, E. D., Thayer, M. L. \& Kirkwood, S. (1979) J. Biol. Chem. 254, 11399-11404

Eckfeldt, J. H. \& Yonetani, T. (1976) Arch. Biochem. Biophys. 173, 273-281

Feldman, R. I. \& Weiner, H. (1972) J. Biol. Chem. 241, 267-272

Haldane, J. B. S. (1930) Enzymes, Longmans, Green and Co., London

Harris, J. I. \& Waters, M. (1976) Enzymes 3rd Ed. 13 , $1-49$

Hart, G. J. \& Dickinson, F. M. (1978) Biochem. J. 175 , 753-756

Hart, G. J. \& Dickinson, F. M. (1982) Biochem. J. 203, 617-627

Katz, M. \& Westley, J. (1979) J. Biol. Chem. 254, 91429147

Kitson, T. M. (1978) Biochem. J. 175, 83-90

Kitson, T. M. (1981) Biochem. J. 199, 255-258

Kitson, T. M. (1982a) Biochem. J. 203, 743-754

Kitson, T. M. (1982b) Biochem. J. 207, 81-89

MacGibbon, A. K. H., Blackwell, L. F. \& Buckley, P. D. (1977) Eur. J. Biochem. 77, 93-100

MacGibbon, A. K. H., Haylock, S. J., Buckley, P. D. \& Blackwell, L. F. (1978) Biochem. J. 171, 533-538

Sakakibara, R., Kitajima, S., Hartman, F. C. \& Uyeda, K. (1984) J. Biol. Chem. 259, 14023-14028

Sidhu, R. S. \& Blair, A. H. (1975a) J. Biol. Chem. 250, 7894-7898

Sidhu, R. S. \& Blair, A. H. (1975b) J. Biol. Chem. 250, 7899-7904 